\title{
Lewis acid catalyzed $N$-acylation of carbamates and oxazolidinones
}

\author{
Chada Raji Reddy, Bodugam Mahipal, and Srinivasa Rao Yaragorla \\ Organic Division-I, Indian Institute of Chemical Technology, Hyderabad-500007, India \\ E-mail: rajireddy@iict.res.in
}

\begin{abstract}
The reaction of various carbamates and oxazolidinones with carboxylic acid anhydrides in presence of Lewis acid catalysis is described. The $N$-acylation was effectively promoted by catalytic amount of $\mathrm{ZnCl}_{2}$ to produce the corresponding $\mathrm{N}$-acyl products in good yields under solvent-free conditions. Carboxylic acids were also successfully used as acylating agents via mixed anhydride method.
\end{abstract}

Keywords: Acylation, carbamates, oxazolidinones, Lewis acid, zinc (II) chloride

\section{Introduction}

$N$-Acyl carbamates and oxazolidinones are important synthetic building blocks towards synthesis of bio-active molecules. ${ }^{1,} 2$ Furthermore, $N$-acyl oxazolidinones have found extensive applications in the asymmetric synthesis as chiral auxiliaries. ${ }^{3}$ The established methods to obtain these $\mathrm{N}$-acyl compounds generally requires $\mathrm{NH}$ activation of amide and/or acyl donor, due to the less basic nature of nitrogen atom in carbamates and oxazolidinones compared to the amines. The methods include the reaction of carbamates and oxazolidinones with acid chlorides or anhydrides in basic reaction conditions using the bases such as trialkyl amines, pyridines etc. ${ }^{2 \mathrm{i}, 4}$ and others. ${ }^{5}$ The $\mathrm{N}$-acylation of these less nucleophilic compounds has not received considerable attention under the acidic medium. To our knowledge, only few reports in the literature is described. ${ }^{6}$ However, strong acidic conditions viz. conc. $\mathrm{H}_{2} \mathrm{SO}_{4}$ or $\mathrm{HBr} / \mathrm{AcOH}$ as well as elevated temperatures are required to achieve such conversions. Recently, $N$-acylation of amides/oxazolidinones with acid anhydrides was demonstrated by the dual activation using $\mathrm{MgBr}_{2} \cdot \mathrm{OEt}_{2}{ }^{7}$ Nevertheless, 2 equiv. of $\mathrm{MgBr}_{2} \cdot \mathrm{OEt}_{2}$ and 3 equiv. of $\mathrm{Et}_{3} \mathrm{~N}$ were used for the reaction. Hence, the development of new methods using Lewis acids in catalytic amounts is of practical importance. In continuation of our interest in Lewis acid catalyzed transformations ${ }^{8,9}$ herein, we report a facile synthesis of $\mathrm{N}$-acyl carbamates and oxazolidinones in the presence of Lewis acid catalyst (Scheme 1). 


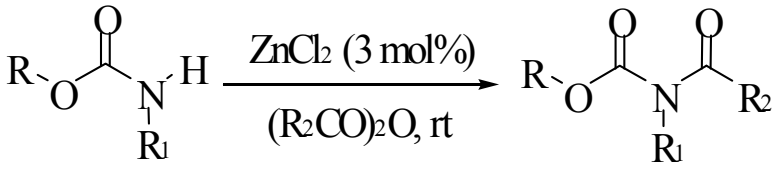

$\mathrm{R}=$ alkyl, aryl; $\mathrm{R} \mathrm{l}=\mathrm{H}$, alkyl or

$\mathrm{R}=\mathrm{R} 1=-\mathrm{CH}_{2}-\mathrm{CHBn}-$

$\mathrm{R} 2=\mathrm{Me}, \mathrm{Et},{ }^{n} \mathrm{Pr},{ }^{\mathrm{B}} \mathrm{Bu}, \mathrm{Ph}$

\section{Scheme 1}

\section{Results and Discussion}

In order to find the suitable conditions, the $N$-acetylation of benzyl carbamate 1a with acetic anhydride was performed at room temperature in the presence of different Lewis acid catalysts under solvent-free conditions. All the catalysts used i.e. $\mathrm{ZnCl}_{2}, \mathrm{FeCl}_{3}, \mathrm{MoCl}_{5}, \mathrm{~B}\left(\mathrm{C}_{6} \mathrm{~F}_{5}\right)_{3}$ and $\mathrm{I}_{2}$ were found to be effective towards the $N$-acylation of benzyl carbamate (entries 1 to 5 , Table 1). No desired product was obtained when the reaction was carried out in the absence of catalyst even after $12 \mathrm{~h}$ (entry 6, Table 1). Although, most of these Lewis acid provided good to better yields, zinc(II) chloride, for obvious reasons (being cheapest, milder conditions and easy to handle) was chosen for further experiments.

Table 1. Catalytic activity of various Lewis acids for the $N$-acetylation of benzyl carbamate with acetic anhydride ${ }^{\mathrm{a}}$

\begin{tabular}{cccc}
\hline entry & $\begin{array}{c}\text { Lewis acid } \\
(3 \mathrm{~mol} \%)\end{array}$ & $\begin{array}{c}\text { time } \\
(\mathrm{min})\end{array}$ & ${\text { yield }(\%)^{\mathrm{b}}}$ \\
\hline 1 & $\mathrm{ZnCl}_{2}$ & 5 & 95 \\
2 & $\mathrm{FeCl}_{3}$ & 3 & 94 \\
3 & $\mathrm{MoCl}_{5}$ & 5 & 92 \\
4 & $\mathrm{~B}\left(\mathrm{C}_{6} \mathrm{~F}_{5}\right)_{3}$ & 10 & 92 \\
5 & $\mathrm{I}_{2}$ & 15 & 85 \\
6 & - & 720 & - \\
\hline
\end{tabular}

${ }^{a}$ All the reactions were carried out at room temperature under solvent free conditions. ${ }^{b}$ Isolated yields after column purification

As a first example, benzyl carbamate $\mathbf{1 a}\left(\mathrm{CbzNH}_{2}\right)$ was treated with acetic anhydride in presence of $3 \mathrm{~mol} \%$ of $\mathrm{ZnCl}_{2}$ under solvent-free conditions to afford the $\mathrm{N}$-acetyl benzyl 
carbamate 2a in 95\% yield (entry 1, Table 2). Successful attempts were also taken to acylate 1a with other acid anhydrides, propionic, pivalic and benzoic anhydrides under similar reaction conditions (entries 2 to 4, Table 2). Phenyl carbamate $\mathbf{1 b}$, furnished the corresponding $N$-acetyl product $\mathbf{2 e}$ in $95 \%$ yield (entry 5, Table 2). However, tert-butyl carbamate $\mathbf{1 c}\left(\mathrm{BocNH}_{2}\right)$ failed to furnish the desired product $\mathbf{2 f}$ (entry 6, Table 2). Certain secondary carbamates including aminoacid carbamate $1 \mathbf{d}$ and amine carbamate $1 \mathrm{e}$ were also studied for $\mathrm{N}$-acetylation and was found to provide the acylated desired products $\mathbf{2 g}$ and $\mathbf{2 h}$ in $93 \%$ and $94 \%$ yields, respectively (entries 7 and 8 , Table 2). Then after, we have further highlighted this Lewis acid catalyzed reaction by extending this method for $N$-acylation of oxazolidinones to produce important constructive products in asymmetric synthesis. Accordingly, oxazolidinone $\mathbf{1 f}$ and 1,3-oxazolidine-2-thione $1 \mathrm{~g}$ were treated with different anhydrides in the presence of $3 \mathrm{~mol} \% \mathrm{ZnCl}_{2}$ to obtain the corresponding $N$-acylated products $\mathbf{2 i}$ to $\mathbf{2 k}$ in good yields (entries 9 to 11, Table 2). The formation of corresponding acylated products $\mathbf{2 g}$ to $\mathbf{2 k}$ from $\mathbf{1 d}$ to $\mathbf{1 g}$ respectively was observed without any racemization (entries 7 to 11 , Table 2).

Encouraged by the above results, we have further investigated the possibility of using carboxylic acids as acylating agents for the $\mathrm{N}$-acylation of carbamates under the Lewis acid catalysis for the first time. Among the anhydrides studied, benzoic anhydride was found to be the least reactive (entry 4, Table 2). Therefore, we have decided to perform the $N$-acylation of carbamate with carboxylic acid in presence of benzoic anhydride by adopting the mixed anhydride method. ${ }^{10}$ Consequently, the reaction of phenyl acetic acid $\mathbf{3 a}$ with benzyl carbamate $1 \mathrm{a}$ in presence of benzoic anhydride and $5 \mathrm{~mol} \% \mathrm{ZnCl}_{2}$ in $\mathrm{CH}_{2} \mathrm{Cl}_{2}$ at room temperature for $1.5 \mathrm{~h}$ afforded the corresponding product $\mathbf{4 a}$ in $85 \%$ yield (entry 1, Table 3 ). Phenyl carbamate $\mathbf{1 b}$ was acylated to $\mathbf{4 b}$ by using the same carboxylic acid 3a under the similar conditions. Likewise, the other carboxylic acids $\mathbf{3 b}$ and $\mathbf{3 c}$ were also successfully participated in acylation reaction with carbamates 1a and $\mathbf{1 b}$ to give the corresponding acyl carbamates $\mathbf{4 c}$ to $\mathbf{4 e}$ (entries 3 to 5, Table 3 . Attempts were also made to $N$-acylate the oxazolidinone 1f with carboxylic acid 3a via mixed anhydride method in presence of $\mathrm{ZnCl}_{2}(3 \mathrm{~mol} \%)$, but without success. ${ }^{11}$

\section{Conclusions}

In summary, we have demonstrated a Lewis acid catalyzed method for the $N$-acylation of carbamates and oxazolidinones with acid anhydrides under solvent-free reaction conditions for the first time. The method was effectively extended to use the carboxylic acids as acylating agents. The simple procedure, mild reaction conditions and high yields of the products makes this methodology attractive for applications in organic synthesis. 
Table 2. $\mathrm{ZnCl}_{2}$-Catalyzed $\mathrm{N}$-acylation of carbamates and oxazolidinones

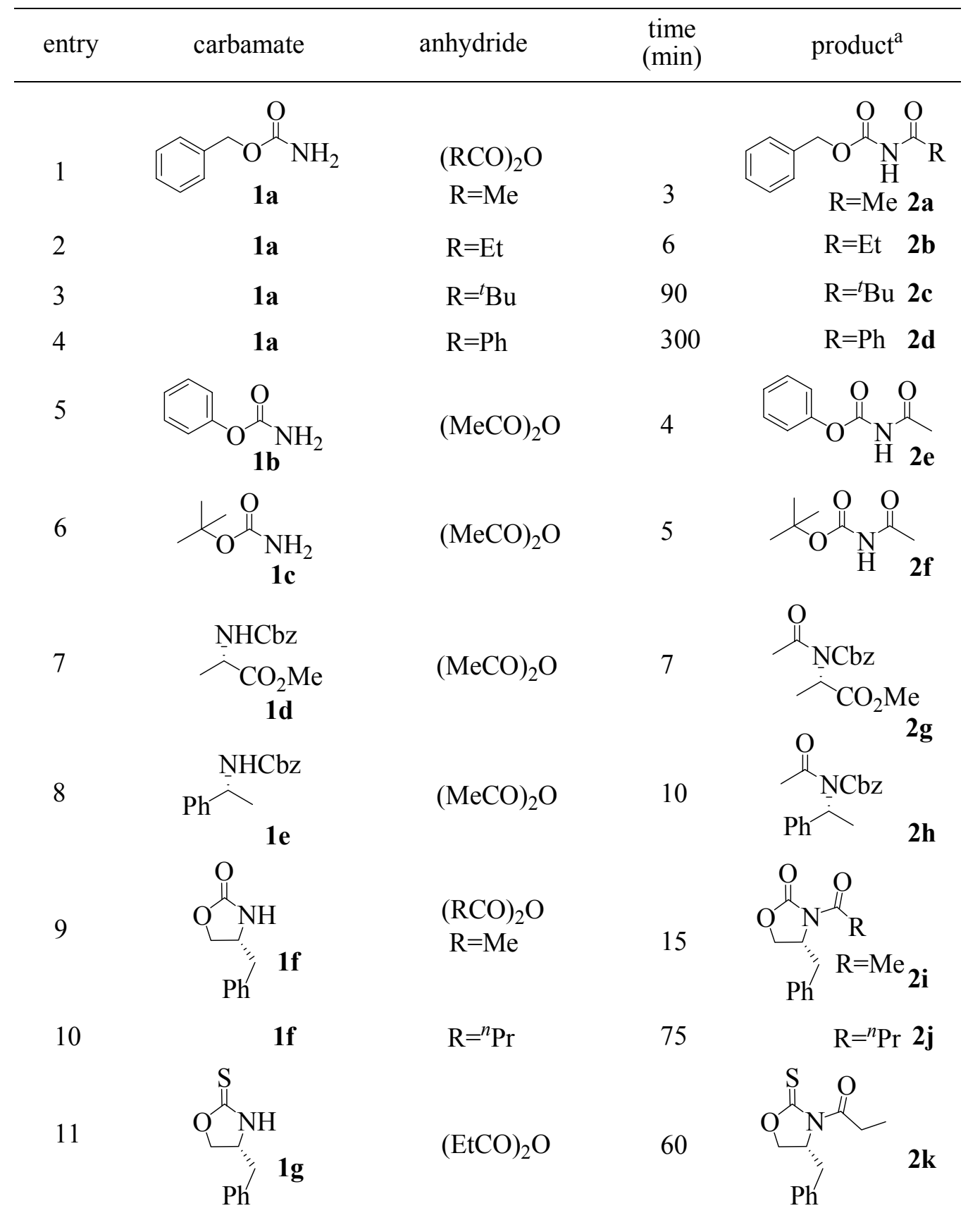

${ }^{a}$ All the products were characterized by ${ }^{1} \mathrm{H}$ NMR, mass and IR spectra. ${ }^{b}$ Isolated yields. ${ }^{c} \mathrm{CH}_{2} \mathrm{Cl}_{2}$ was used as solvent. ${ }^{\mathrm{d}}$ Complex mixture was formed (based TLC). 
Table 3. $N$-Acylation of carbamates using carbocylic acids ${ }^{\mathrm{a}}$

entry $\begin{gathered}\begin{array}{c}\text { carboxylic } \\ \text { acid }\end{array} \\ \text { carbamate }\end{gathered}$

${ }^{\mathrm{a}}$ Reaction conditions: $5 \mathrm{~mol} \% \mathrm{ZnCl}_{2}, 1.2$ equiv $(\mathrm{PhCO})_{2} \mathrm{O}, \mathrm{CH}_{2} \mathrm{Cl}_{2}$, rt. ${ }^{\mathrm{b}}$ All the products were characterized by ${ }^{1} \mathrm{H}$ NMR, mass and IR spectra. ${ }^{\mathrm{C}}$ Isolated yields.

\section{Experimental Section}

General Procedures. The chemicals such as carbamates, anhydrides, oxazolidinone, carboxylic acids and all the Lewis acid catalysts were purchased from Aldrich and used as received. Dichloromethane was distilled over calcium hydride. The optical rotations were recorded using a Jasco Dip 360 digital polarimeter. IR spectra were recorded on Perkin-Elmer 683 spectrometer. ${ }^{1} \mathrm{H}$ NMR (200 MHz and $300 \mathrm{MHz}$ ) and ${ }^{13} \mathrm{C}$ NMR (75 MHz) spectra of samples in $\mathrm{CDCl}_{3}$ were recorded on Bruker Avance spectrometer. Chemical shifts were reported in ppm with respect to internal TMS. Coupling constants $(J)$ are quoted in Hz. Mass spectra were obtained on an Agilent Technologies LC/MSD Trap SL.

\section{General experimental procedure for $N$-acylation of carbamates and oxazolidinones with anhydrides (for products 2 a to $2 k$ )}

To a mixture of carbamte or oxazolidinone $(1.0 \mathrm{mmol})$ and anhydride $(1.5 \mathrm{mmol}), 3 \mathrm{~mol} \%$ of anhydrous $\mathrm{ZnCl}_{2}$ was added and the reaction stirred for the given time (see Table 2). The reaction mixture was diluted with dichloromethane $(15 \mathrm{~mL})$ and washed with water $(10 \mathrm{~mL})$ and brine $(10 \mathrm{~mL})$ solution. The organic layers were dried over $\mathrm{Na}_{2} \mathrm{SO}_{4}$ and evaporated in vacuo. The crude compound was purified by column chromatography (hexanes and ethyl acetate) to afford the corresponding $\mathrm{N}$-acylated product. 
Benzyl propionylcarbamate (2b). White powder, m.p. $119-121{ }^{\circ} \mathrm{C}$; ${ }^{1} \mathrm{H}$ NMR $(200 \mathrm{MHz}$, $\left.\mathrm{CDCl}_{3}\right): \delta 7.60($ br s, $1 \mathrm{H}), 7.39-7.34(\mathrm{~m}, 5 \mathrm{H}), 5.18(\mathrm{~s}, 2 \mathrm{H}), 2.79(\mathrm{q}, J=7.4 \mathrm{~Hz}, 2 \mathrm{H}), 1.16(\mathrm{t}, J=$ $7.4 \mathrm{~Hz}, 3 \mathrm{H}) ;{ }^{13} \mathrm{C} \mathrm{NMR}\left(75 \mathrm{MHz}, \mathrm{CDCl}_{3}\right): \delta 175.7,151.9,135.2,128.84,128.80,128.4,67.9$, 29.7, 8.4; IR(KBr): v 3261, 3187, 1756, 1696, 1526, 1195, $1048 \mathrm{~cm}^{-1}$; EIMS: $\mathrm{m} / z 230.0$ (M+Na).

Benzyl pivaloylcarbamate (2c). White solid, m.p. 103-105 ${ }^{\circ} \mathrm{C} ;{ }^{1} \mathrm{H} \mathrm{NMR}\left(200 \mathrm{MHz}, \mathrm{CDCl}_{3}\right): \delta$ 7.70 (br s, 1H), 7.44-7.32 (m, 5H), $5.20(\mathrm{~s}, 2 \mathrm{H}), 1.23(\mathrm{~s}, 9 \mathrm{H}) ;{ }^{13} \mathrm{C}$ NMR $\left(75 \mathrm{MHz}, \mathrm{CDCl}_{3}\right)$ : $\delta 175.8,151.0,135.2,128.74,128.72,67.8,40.3,27.0$; IR (KBr): v 3296, 2964, 1772, 1687, 1515, 1209, 1165, $700 \mathrm{~cm}^{-1}$; HRMS-ESI Calcd for $\mathrm{C}_{13} \mathrm{H}_{17} \mathrm{NO}_{3} \mathrm{Na}$ : 258.1109; Found: 258.1106 .

Phenyl acetylcarbamate (2e). White powder, m.p. 120-122 ${ }^{\circ} \mathrm{C} ;{ }^{1} \mathrm{H}$ NMR $\left(200 \mathrm{MHz}, \mathrm{CDCl}_{3}\right): \delta$ 8.10 (br s, 1H), $7.46-7.35$ (m, 2H), 7.33 - $7.22(\mathrm{~m}, 1 \mathrm{H}), 7.20-7.12(\mathrm{~m}, 2 \mathrm{H}), 2.49(\mathrm{~s}, 3 \mathrm{H}) ;{ }^{13} \mathrm{C}$ NMR $\left(75 \mathrm{MHz} \mathrm{CDCl}_{3}\right): \delta 173.1,150.9,149.9,129.7,126.5,121.6,24.3$; IR (KBr): v 3257, 3197, 1769, 1704, 1531, 1483, 1220, 1117, $729 \mathrm{~cm}^{-1}$; HRMS-ESI Calcd for $\mathrm{C}_{9} \mathrm{H}_{9} \mathrm{NO}_{3} \mathrm{Na}$ : 202.0475; Found: 202.0480.

(S)-Methyl 2-( $N$-(benzyloxycarbonyl) acetamido) propa-noate $(\mathbf{2 g})$. Colorless liquid; $[\alpha]_{\mathrm{D}}{ }^{25}=$ -14.6 (c 1 in $\left.\mathrm{CHCl}_{3}\right)$; ${ }^{1} \mathrm{H}$ NMR $\left(200 \mathrm{MHz}, \mathrm{CDCl}_{3}\right): \delta$ 7.40-7.34 (m, 5H), 5.38- $5.13(\mathrm{~m}, 3 \mathrm{H}), 3.53$ $(\mathrm{s}, 3 \mathrm{H}), 2.54(\mathrm{~s}, 3 \mathrm{H}), 1.48(\mathrm{~s}, 2 \mathrm{H}), 1.45(\mathrm{~s}, 1 \mathrm{H}) ;{ }^{13} \mathrm{C} \mathrm{NMR}\left(75 \mathrm{MHz}, \mathrm{CDCl}_{3}\right): \delta 172.3,171.2$, 153.6, 134.6, 128.9, 128.8, 128.7, 69.0. 52.3, 51.9, 26.6, 15.5 ; IR (neat): v 3457, 2949, 1745, 1703, 1435, 1385, 1252, $698 \mathrm{~cm}^{-1}$; HRMS-ESI Calcd for $\mathrm{C}_{14} \mathrm{H}_{17} \mathrm{NO}_{5} \mathrm{Na}$ : 302.1011; Found: 302.1004 .

(R)-Benzyl acetyl(1-phenylethyl)carbamate (2h). Colorless liquid; $[\alpha]_{\mathrm{D}}{ }^{25}=-55.5$ (c 1.5 $\left.\mathrm{CHCl}_{3}\right) ;{ }^{1} \mathrm{H}$ NMR $\left(200 \mathrm{MHz}, \mathrm{CDCl}_{3}\right): \delta$ 7.44-7.12 (m, 8H), 7.10-6.95 (m, 2H), 6.18-5.97 (q, $J=$ $6.6 \mathrm{~Hz}, 1 \mathrm{H}), 5.10-4.97(\mathrm{dd}, J=6.4 \mathrm{~Hz}, J=13.6 \mathrm{~Hz}, 2 \mathrm{H}), 2.53(\mathrm{~s}, 3 \mathrm{H}), 1.65(\mathrm{~d}, J=7.3 \mathrm{~Hz}, 3 \mathrm{H})$; ${ }^{13} \mathrm{C}$ NMR $\left(75 \mathrm{MHz}, \mathrm{CDCl}_{3}\right): \delta 173.1,154.8,141.3,134.7,128.6,128.5,128.3,126.8,126.5$, 68.5, 51.6, 27.0, 17.2.; IR (KBr): v 3032, 2944, 1734, 1695, 1239, $697 \mathrm{~cm}^{-1}$; HRMS-ESI Calcd for $\mathrm{C}_{18} \mathrm{H}_{19} \mathrm{NO}_{3} \mathrm{Na}$ : 320.1262; Found: 320.1275. 2a, ${ }^{12} \mathbf{2 d},{ }^{12} \mathbf{2} \mathbf{i},{ }^{13} \mathbf{2 j}^{14}$ and $\mathbf{2} \mathbf{k}^{15}$ : IR, ${ }^{1} \mathrm{H},{ }^{13} \mathrm{C}$ NMR and mass spectral data of these known compounds were identical with the reported data

General experimental procedure for $\mathrm{N}$-acylation of carbamates with carboxylic acids (for products 4 a to 4 e)

To a stirred solution of $5 \mathrm{~mol} \%$ anhydrous $\mathrm{ZnCl}_{2}$ in anhydrous dichloromethane $(10 \mathrm{~mL})$, carboxylic acid $(1.2 \mathrm{mmol})$ was added followed by the addition of benzoic anhydride $(1.2 \mathrm{mmol})$ under a nitrogen atmosphere at room temperature. After $10 \mathrm{~min}$, a solution of carbamate in $\mathrm{CH}_{2} \mathrm{Cl}_{2}$ was added and the resulting reaction mixture was stirred for the given time (see Table 3). The reaction mixture was diluted with dichloromethane $(20 \mathrm{~mL})$ and washed with water $(10 \mathrm{~mL})$ then brine $(10 \mathrm{~mL})$ and the organic layers were dried over $\mathrm{Na}_{2} \mathrm{SO}_{4}$, and evaporated in vacuo. The crude compound was purified by column chromatography (hexanes and ethyl acetate) to afford the corresponding $N$-acylated product.

Benzyl 2-phenylacetylcarbamate (4a). White solid, m.p. $128-130{ }^{\circ} \mathrm{C} ;{ }^{1} \mathrm{H}$ NMR (200 MHz, $\left.\mathrm{CDCl}_{3}\right): \delta 8.10$ (br s, $\left.1 \mathrm{H}\right), 7.38-7.17(\mathrm{~m}, 10 \mathrm{H}), 5.16(\mathrm{~s}, 2 \mathrm{H}), 4.05(\mathrm{~s}, 2 \mathrm{H}) ;{ }^{13} \mathrm{C} \mathrm{NMR}(75 \mathrm{MHz}$, $\left.\mathrm{CDCl}_{3}\right): \delta 172.5,151.7,135.0,133.6,133.5,129.5,128.8,128.6,127.5,127.4,68.1,42.9$; IR 
(KBr): v 3255, 3172, 1758, 1693, 1523, 1220, $702 \mathrm{~cm}^{-1}$; HRMS-ESI Calcd for $\mathrm{C}_{16} \mathrm{H}_{15} \mathrm{NO}_{3} \mathrm{Na}$ : 292.0949; Found: 292.0955.

Phenyl 2-phenylacetylcarbamate (4b). Semi solid; ${ }^{1} \mathrm{H}$ NMR $\left(200 \mathrm{MHz}, \mathrm{CDCl}_{3}\right): \delta 7.70$ (br s, $1 \mathrm{H}), 7.42-7.14(\mathrm{~m}, 8 \mathrm{H}), 7.07(\mathrm{~d}, J=7.3 \mathrm{~Hz}, 2 \mathrm{H}), 4.07(\mathrm{~s}, 2 \mathrm{H}) \cdot ;{ }^{13} \mathrm{C} \mathrm{NMR}\left(75 \mathrm{MHz}, \mathrm{CDCl}_{3}\right): \delta$ 172.1, 150.2, 150.0, 133.3, 129.8 (2C), 128.9, 127.6, 126.6, 121.5, 42.9.; IR (KBr): v 3256, 2925, 1760, 1533, 1486, 1186, $804 \mathrm{~cm}^{-1}$; HRMS-ESI Calcd for $\mathrm{C}_{15} \mathrm{H}_{13} \mathrm{NO}_{3} \mathrm{Na}$ : 278.0793; Found: 278.0798 .

Benzyl 2-(4-isobutylphenyl)propanoylcarbamate (4c). Light yellowish solid, m.p. 124-126 ${ }^{\circ} \mathrm{C}$; ${ }^{1} \mathrm{H}$ NMR $\left(200 \mathrm{MHz}, \mathrm{CDCl}_{3}\right.$ ): $\delta 7.36$ (br s, $\left.1 \mathrm{H}\right), 7.37-7.06$ (m, 9H), 5.12 (s, 2H), 4.30-4.12 (q, $J=$ $6.6 \mathrm{~Hz}, 1 \mathrm{H}), 2.45(\mathrm{~d}, J=7.3 \mathrm{~Hz}, 2 \mathrm{H}), 1.97-1.74(\mathrm{~m}, 1 \mathrm{H}), 1.48(\mathrm{~d}, J=6.6 \mathrm{~Hz}, 3 \mathrm{H}), 0.89(\mathrm{~d}, J=$ $6.6 \mathrm{~Hz}, 6 \mathrm{H}) \cdot ;{ }^{13} \mathrm{C} \mathrm{NMR}\left(75 \mathrm{MHz}, \mathrm{CDCl}_{3}\right): \delta 174.1,151.0,141.2,137.2,135.1,129.8,128.8$, 128.5, 128.4, 127.7, 67.9, 45.9, 45.2, 30.3, 22.5, 18.8.; IR (KBr): v 3256, 2925, 1750, 1541, 1517, 1238. $697 \mathrm{~cm}^{-1}$; HRMS-ESI Calcd for $\mathrm{C}_{21} \mathrm{H}_{25} \mathrm{NO}_{3} \mathrm{Na}$ : 362.1732; Found: 362.1724 .

Phenyl 2-(4-isobutylphenyl)propanoylcarbamate (4d). White powder, m.p. $80-82{ }^{\circ} \mathrm{C} ;{ }^{1} \mathrm{H}$ NMR (200 MHz, $\left.\mathrm{CDCl}_{3}\right): \delta 8.20-8.08(\mathrm{~m}, 2 \mathrm{H}), 8.00$ (br s, $\left.1 \mathrm{H}\right), 7.71-7.55(\mathrm{~m}, 1 \mathrm{H}), 7.56-7.31$ (m, 2H), 7.30-7.19 (m, 2H), 7.18-7.05 (m, 2H), $3.76(\mathrm{q}, J=6.8 \mathrm{~Hz}, 1 \mathrm{H}), 2.46(\mathrm{~d}, J=7.0 \mathrm{~Hz}, 2 \mathrm{H})$, 1.96-1.74 (m, 1H), $1.54(\mathrm{dd}, J=7.0,2.3 \mathrm{~Hz}, 3 \mathrm{H}), 0.91(\mathrm{~d}, J=6.2 \mathrm{~Hz}, 6 \mathrm{H}) ;{ }^{13} \mathrm{C}$ NMR $(75 \mathrm{MHz}$, $\left.\mathrm{CDCl}_{3}\right): \delta 174.8,149.9,149.7,141.3,137.1,129.8,129.7,127.8,127.4,126.4,121.5,45.7,45.2$, 30.3, 22.6, 19.0; IR(KBr): $v 3263,2955,1762,1704,1456,1287 \mathrm{~cm}^{-1}$; HRMS-ESI Calcd for $\mathrm{C}_{20} \mathrm{H}_{24} \mathrm{NO}_{3}$ : 326.1749; Found: 326.1756 .

Phenyl hex-5-ynoylcarbamate (4e). White solid, m.p. $74-76{ }^{\circ} \mathrm{C} ;{ }^{1} \mathrm{H}$ NMR $\left(200 \mathrm{MHz}, \mathrm{CDCl}_{3}\right): \delta$ $7.80($ br s, $1 \mathrm{H}), 7.52-7.09(\mathrm{~m}, 5 \mathrm{H}), 2.98(\mathrm{t}, J=7.3 \mathrm{~Hz}, 2 \mathrm{H}), 2.38-2.26(\mathrm{~m}, 2 \mathrm{H}), 2.02-1.84(\mathrm{~m}$, $3 \mathrm{H}) . ;{ }^{13} \mathrm{C} \mathrm{NMR}\left(75 \mathrm{MHz}, \mathrm{CDCl}_{3}\right): \delta 174.0,150.4,150.0,129.8,126.6,121.6,83.5,69.4,35.0$, 22.9, 17.9.; IR (KBr): v 3426, 3271, 2924, 1758, 1525, 1146, $658 \mathrm{~cm}^{-1}$.; HRMS-ESI Calcd for $\mathrm{C}_{13} \mathrm{H}_{13} \mathrm{NO}_{3} \mathrm{Na}: 254.0793$; Found: 254.0796.

\section{Acknowledgements}

BM and YSR thank the CSIR, New Delhi for financial assistance. CRR is greatful to Dr. S. Chandrasekhar and Dr. J. S. Yadav for their encouragement and support.

\section{References and Notes}

1. Gardner, T. S.; Wenis, E.; Lee, J. J. Org. Chem. 1954, 19, 753. (b) Fraser, J.; Clinch, P. G.; Reay, R. C. J. Sci. Fd Agric. 1965, 16, 615. (c) Fahmy, M. A. H.; Fukuto, T. R. J. Econ. Ent. 1970, 63, 1783. (d) D'Silva, T. D. J. U.S. 4568671, 1986, 8 pp. (e) Liu, W.; Sheppeck, J. E., II; Colby, D. A.; Huang, H.-B.; Nairn, A. C.; Chamberlin, A. R. Bioorg. Med. Chem. Lett. 2003, 13, 1597. 
2. Brouillette, W. J.; Smissman, E. E.; Grunewald, G. L. J. Org. Chem. 1979, 44, 839. (b) Marron, T. G.; Roush, W. R. Tetrahedron Lett. 1995, 36, 1581. (c) Gaudl, K.-U.; Lachowicz, A.; Grahe, G. F. Ger. Offen. DE 19523175, 1997, 8 pp. (d) Ciclosi, M.; Fava, C.; Galeazzi, R.; Orena, M.; Sepulveda-Arques, J. Tetrahedron Lett. 2002, 43, 2199. (e) Ling, T.; Chowdhury, C.; Kramer, B. A.; Vong, B. G.; Palladino, M. A.; Theodorakis, E. A. J. Org. Chem. 2001, 66, 8843. (f) Bouzide, A.; Sauve, G. Tetrahedron Lett. 2002, 43, 1961. (g) Liu, S.; Fan, Y.; Peng, X.; Wang, W.; Hua, W.; Akber, H.; Liao, L. Tetrahedron Lett. 2006, 47, 7681. (h) Peters, R.; Althaus, M.; Diolez, C.; Rolland, A.; Manginot, E.; Veyrat, M. J. Org. Chem. 2006, 71, 7583. (i) Chen, Z.-L.; Zhou, W.-S. Tetrahedron Lett. 2006, 47, 5289.

3. Evans, D. A.; Ennis, M. D.; Mathre, D. J. J. Am. Chem. Soc. 1982, 104, 1737. (b) Evans, D. A. Aldrichimica Acta 1982, 15, 23. (c) Ager, D. J.; Prakash, I.; Shaad, D. R. Chem. Rev. 1996, 96, 835. (d) Furuno, H.; Inoue, T.; Abiko, A. Tetrahedron Lett. 2002, 43, 8297. (e) Crimmins, M. T.; King, B. W.; Tabet, E. A.; Chaudhary, K. J. Org. Chem. 2001, 66, 894.

4. (a) Roush, W. R; Pfeifer, L. A. J. Org. Chem. 1998, 63, 2062. (b) Ager, D. J.; Allen, D. R.; Schaad, D. R. Synthesis 1996, 1283. (c) Schelkun, R. M.; Yaen, P.-W.; Wustrow, D. J.; Kinsora, J.; Su, T.-Z.; Vartanian, M. G. Bioorg. Med. Chem. Lett. 2006, 16, 2329; (d) Marigo, M.; Schulte, T.; Franzen, J.; Jorgensen, K. A. J. Am. Chem. Soc. 2005, 127, 15710. (e) Wei, P.; Kerns, R. J. Tetrahedron Lett. 2005, 46, 6901. (f) Hein, J. E.; Hultin, P. G. Tetrahedron: Asymmetry 2005, 16, 2341. (g) Crimmins, M. T.; Chaudhary, K. Org. Lett. 2000, 2, 775.

5. (a) Ben-Ishai; Katchalski; J. Org. Chem. 1951, 16, 1025. (b) Seiller, B.; Heins, D.; Bruneau, C.; Dixneuf, P. H. Tetrahedron 1995, 51, 10901.

6. Ferrari, G.; Casagrande, C. Farmaco, Ed.. Sci. 1963, 18, 780. (b) Miyake, T.; Tsuchiya, T.; Umezawa, S.; Saito, S.; Umezawa, H. Bull. Chem. Soc. Jpn. 1986, 59, 1387. (c) Thom, C.; Kocienski, P. Synthesis 1992, 582.

7. Yamada, S.; Yaguchi, S.; Matsuda, K. Tetrahedron Lett. 2002, 43, 647.

8. Reddy, Ch. R.; Mahipal, B.; Yaragorla, S. R. Tetrahedron Lett. 2007, 48, 7528.

9. (a) Reddy, Ch. R.; Madhavi, P. M.; Reddy A. S. Tetrahedron Lett. 2007, 48, 7169. (b) Chandraekhar, S.; Basu, D.; Reddy, Ch. R. Synthesis 2007, 1509.

10. Chen, C.-T.; Kuo, J.-H.; Pawar, V. D.; Munot, Y. S.; Weng, S.-S.; Ku, C.-H.; Liu, C.-Y. J. Org. Chem. 2005, 70, 1188.

11. The reaction did not proceed even at higher temperatures and for longer reaction time.

12. Shangguan, N.; Katukojvala, S.; Greenberg, R.; Williams, L. J. J. Am. Chem. Soc. 2003, $125,7754$.

13. Ager, D. J.; Allen, D. R.; Schaad, D. R. Synthesis 1996, 11, 1283.

14. (a) Tao, T.; Parry, R. J. Org. Lett. 2001, 19, 3045. (b) Evans, D. A.; Rieger, D. L.; Jones, T. K.; Kaldor, S, W. J. Org. Chem. 1990, 55, 6260.

15. Michael, T. C.; Bryan, W. K.; Elie, A. T.; Kleem, C. J. Org. Chem. 2001, 66, 894. 